\title{
Scaling properties in large assemblies of simple dynamical units driven by long-wave random forcing
}

$\operatorname{AUTHOR}(S):$

Kuramoto, Y; Nakao, H

\section{CITATION:}

Kuramoto, Y...[et al]. Scaling properties in large assemblies of simple dynamical units driven by long-wave random forcing. PHYSICAL REVIEW LETTERS 1997, 78(21): $4039-4042$

\section{ISSUE DATE:}

1997-05-26

URL:

http://hdl.handle.net/2433/50551

RIGHT:

Copyright 1997 American Physical Society 


\title{
Scaling Properties in Large Assemblies of Simple Dynamical Units Driven by Long-Wave Random Forcing
}

\author{
Yoshiki Kuramoto and Hiroya Nakao \\ Department of Physics, Graduate School of Sciences, Kyoto University, Kyoto 606, Japan
}

(Received 14 January 1997)

\begin{abstract}
Large assemblies of nonlinear dynamical units driven by a long-wave fluctuating external field are found to generate strong turbulence with scaling properties. This type of turbulence is so robust that it persists over a finite parameter range with parameter-dependent exponents of singularity, and is insensitive to the specific nature of the dynamical units involved. Whether or not the units are coupled with their neighborhood is also unimportant. It is discovered numerically that the derivative of the original amplitude field exhibits strong spatial intermittency with multifractal structure. [S0031-9007(97)03232-8]
\end{abstract}

PACS numbers: 47.27.-i, 05.45.+b, 47.53.+n, 87.10.+e

We report our discovery of a new type of turbulent behavior which arises generally in large assemblies of simple dynamical units driven by a long-wave randomly fluctuating field. The driving field may actually be a self-generated internal field due to long-range interaction, and this particular situation was studied in a previous paper [1] where a rough explanation of the origin of power-law correlations was also given. The present study thus aims at expanding as much as possible the class of systems capable of exhibiting the same type of turbulence, and also proposing a more transparent and coherent explanation of the phenomena.

An illustrative example is given by an array of uncoupled logistic maps $f(X)=a X(1-X)$ with driving

$$
X_{n+1}(j)=f\left(X_{n}(j)\right)+h_{n}(j), \quad j=1,2, \ldots, N,
$$

where $h_{n}(j)=\frac{K}{2}\left(1+\cos \left[2 \pi\left\{\frac{j}{N}+\psi_{n}\right\}\right]\right), \psi_{n}$ being a random variable in the interval $(0,1]$ with uniform distribution. Were it not for spatial dependence of $h_{n}$, Eq. (1) would represent $N$ identical copies of a randomly driven map; the dynamics of such an ensemble was studied in [2]. Making $h_{n}$ nonuniform changes the problem completely. Let the parameter values be set such that individual maps are entrained to $h_{n}$ in the sense that their maximum Lyapunov exponent (common to all maps) is negative. Unlike $h_{n}$, however, the corresponding amplitude profile is not smooth at all, a typical example of which is displayed in Fig. 1(a). Such ill-behaved nature of the pattern is even amplified in Fig. 1(b) which shows a strongly intermittent pattern of the differential amplitudes $Y(j) \equiv|X(j+1)-X(j)|$ constructed from Fig. 1(a).

Before proceeding to further numerical study, some theoretical predictions will be made as to the statistics of turbulence to be shared by the above system or more general assemblies of units under long-wave random driving. For this purpose, it seems more convenient to work with a picture in which the dynamical units form a quasicontinuum rather than a lattice, and the driving field has a characteristic wavelength of $O(1)$. Our primary concern is to understand how a simple driving field alone can generate a nontrivial correlation between uncoupled units.

Suppose that each unit is represented by a discretetime dynamical system $\boldsymbol{X}_{n+1}=\boldsymbol{f}\left(\boldsymbol{X}_{n}\right)$. Let the units be driven by an additive random force $\boldsymbol{h}_{n}$ which is smooth in space and statistically invariant with respect to spatial translations. The unit at site $\boldsymbol{r}$ is governed by the equation

$$
\boldsymbol{X}_{n+1}(\boldsymbol{r})=\boldsymbol{f}\left(\boldsymbol{X}_{n}(\boldsymbol{r})\right)+\boldsymbol{h}_{n}(\boldsymbol{r}) .
$$

Analogously to fully developed fluid turbulence [3], let us consider various moments of the amplitude increment between two sites. We thus concentrate on a pair of units at sites $\boldsymbol{r}_{0}$ and $\boldsymbol{r}_{0}+\boldsymbol{x}$ with distance $x \equiv|\boldsymbol{x}|$ satisfying $x \ll 1$. The amplitude increment $y_{n}(\boldsymbol{x}) \equiv \boldsymbol{X}_{n}\left(\boldsymbol{r}_{0}+\boldsymbol{x}\right)-$ $\boldsymbol{X}_{n}\left(\boldsymbol{r}_{0}\right)$ obeys the equation

$$
\boldsymbol{y}_{n+1}=\hat{L}_{n} \boldsymbol{y}_{n}+O\left(\left|\boldsymbol{y}_{n}\right|^{2}\right)+\Delta \boldsymbol{h}_{n},
$$

where $\hat{L}_{n} \boldsymbol{y}_{n}$ is the linearization of $\boldsymbol{f}(\boldsymbol{X})$ about $\boldsymbol{X}=$ $\boldsymbol{X}_{n}\left(\boldsymbol{r}_{0}\right)$, and $\Delta \boldsymbol{h}_{n} \equiv \boldsymbol{h}_{n}\left(\boldsymbol{r}_{0}+\boldsymbol{x}\right)-\boldsymbol{h}_{n}\left(\boldsymbol{r}_{0}\right)$ is a quantity of
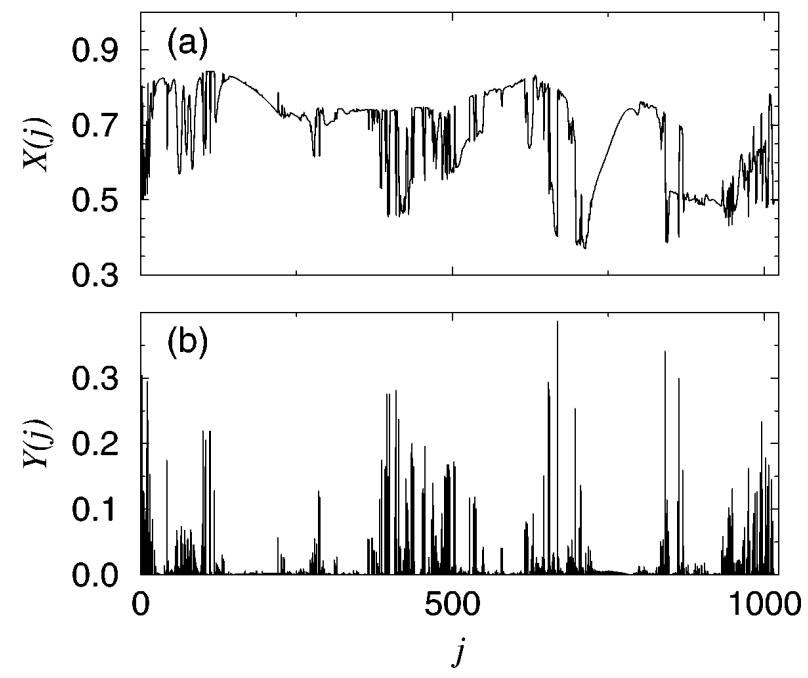

FIG. 1. (a) Instantaneous amplitude profile for the driven logistic maps (1). $N=1024, K=0.2$, and $a=3.7(1-K)$. (b) Profile of differential amplitudes $Y(j)$ constructed from (a). 
$O(x)$. Equation (3) describes a multiplicative stochastic process [4] with small additive noise. Similar equations have recently aroused considerable interest in connection with on-off intermittency and related phenomena [5,6]. While on-off intermittency refers to a certain type of temporal self-similarity peculiar to a special parameter value, our major concern below is a spatial self-similarity observable over an open parameter range.

Equation (3) may be simplified by neglecting all eigenmodes of $\hat{L}_{n}$ other than the least stable one. This leads to a scalar equation for $y_{n} \equiv\left|\mathbf{y}_{n}\right|$

$$
y_{n+1}=e^{\lambda_{n}} y_{n}+O\left(y_{n}^{2}\right)+b_{n} x,
$$

where $\lambda_{n}$ is the local Lyapunov exponent of the unit at site $\mathbf{r}_{0}$, and $b_{n}$ is a randomly changing factor of $O(1)$. For sufficiently small $x$, there is a range of $y$ satisfying $x \ll$ $y \ll 1$ where both the nonlinear and inhomogeneous terms in (4) are negligible. We are thus left with a linear equation $y_{n+1}=e^{\lambda_{n}} y_{n}$ or $z_{n+1}-z_{n}=\lambda_{n}$ in terms of a new variable $z_{n}=\ln y_{n}$. If the random process of $\lambda_{n}$ is Markoffian, which we assume, the probability density $Q_{n}(z)$ for $z_{n}$ evolves in this linear regime according to

$$
Q_{n+1}(z)=\int_{-\infty}^{\infty} w(\lambda) Q_{n}(z-\lambda) d \lambda,
$$

where $w(\lambda)$ is the normalized probability density for $\lambda_{n}$. Equation (5) admits a stationary solution of the form $Q(z) \propto \exp (\beta z) \equiv y^{\beta}$. Thus, the corresponding probability density for $y_{n}$, denoted by $P(y)$, becomes

$$
P(y) \propto y^{-1+\beta},
$$

where $\beta$ is determined as a nontrivial (i.e., nonzero) solution of

$$
\int_{-\infty}^{\infty} e^{-\beta \lambda} w(\lambda) d \lambda=1 .
$$

Note that for sufficiently small $\beta$, we have

$$
\beta=2 \bar{\lambda} / \overline{\lambda^{2}},
$$

where the bar means the average with respect to $w(\lambda)$. We have now to modify (6) by taking into account the effects of the nonlinear and inhomogeneous terms in (4). The nonlinearity, which is assumed to work in such a way that the unstable growth of $y_{n}$ be saturated when $\lambda_{n}>0$, may roughly be incorporated by introducing a cutoff in $P(y)$ at $y=1$. On the other hand, the inhomogeneous term will come into play when $y_{n}$ becomes $O(x)$ or smaller, thus suppressing the power-law divergence of $P(y)$ there. For the purpose of qualitative argument, one may therefore use the following simple model for $P(y)$ :

$$
\begin{gathered}
P(y)=C x^{-1+\beta} \quad(y \leq x), C y^{-1+\beta} \quad(x<y \leq 1), \\
0(y>1), \quad C: \text { normalization const. }
\end{gathered}
$$

This form allows us to calculate the $q$ th moment $\left\langle y(x)^{q}\right\rangle$ for arbitrary $q$. For simplicity, only positive values of $q$ will be considered below. In the subcritical regime $(\beta<$ 0 , i.e., $\bar{\lambda}<0$ ), where the dynamical units are entrained to the driving field, we obtain power-law moments

$$
\left\langle y(x)^{q}\right\rangle \sim x^{q} \quad(q<|\beta|), \quad x^{|\beta|} \quad(q>|\beta|) .
$$

The result of $q$-independent exponent valid for higher moments $(q>|\beta|)$ is anomalous, reflecting strong nonGaussianity of $P(y)$. In the postcritical regime $(\beta>0)$, all moments possess an $x$-independent part, while the residual part still obeys a power law:

$$
\left\langle y(x)^{q}\right\rangle \sim \beta(q+\beta)^{-1}+O\left(x^{\beta}\right) .
$$

The reason why the amplitude difference in the postcritical regime is nonvanishing for vanishing $x$ is that the two units in question have lost their respective synchrony with $h_{n}$, implying also the loss of their mutual synchrony. Note that (10) and (11) are asymptotic formulae valid for $x \rightarrow 0$ under fixed $\beta$. Near $|\beta|=q$ and 0 under fixed $x$, however, there exist crossover regimes (C1) $|(\beta+q) \ln x| \ll 1$ and (C2) $|\beta \ln x| \ll 1$, respectively, in each of which we have $\left\langle y(x)^{q}\right\rangle \sim x^{|\beta|}|\ln x|$ and $|\ln x|^{-1}$.

A few more remarks are now given on the cases of $q=2$ and 1 for which our theory recovers our previous results [1]. We obtain from (10) and (11) the second moment

$$
\begin{gathered}
\left\langle y(x)^{2}\right\rangle \sim x^{2} \quad(-2), \quad x^{|\beta|} \quad(-2<0), \\
\beta(2+\beta)^{-1}+O\left(x^{\beta}\right) \quad(\beta>0),
\end{gathered}
$$

while in the aforementioned crossover regimes, we have $\left\langle y(x)^{2}\right\rangle \sim x^{2}|\ln x|(\mathrm{C} 1)$ and $1 /|\ln x|(\mathrm{C} 2)$.

The case $q=1$ is related to the length of an amplitude versus space curve. This is because the length $S(x)$ for the part of an amplitude profile contained in the unit interval, when measured with the resolution of the minimum length scale $x$, is given by $S(x) \sim x^{-1}\langle y(x)\rangle$. Applying (10) and (11), we thus obtain $S(x) \sim$ const $(\beta<-1), x^{|\beta|-1}(-1<\beta<0)$, and $x^{-1}(\beta>0)$. In the crossover regimes, however, these must be replaced by $S(x) \sim|\ln x|(\mathrm{C} 1)$ and $1 /(x|\ln x|)(\mathrm{C} 2)$. The fractal dimension $D_{f}$ defined by $S(x) \sim x^{1-D_{f}}$ thus becomes

$$
\begin{gathered}
D_{f}=1 \quad(\beta<-1), 2-|\beta| \quad(-1<\beta<0), \\
2(\beta>0),
\end{gathered}
$$

except for the crossover regimes.

The above arguments on discrete-time dynamics can easily be carried over to continuous-time dynamics. One needs only make replacements $n \rightarrow t, n+1 \rightarrow t+$ $d t$, and $\lambda_{n} \rightarrow \lambda(t) d t$. Then, (4) becomes $\dot{y}=\lambda(t) y+$ $O\left(y^{2}\right)+b(t) x$, and (5) reduces to a Fokker-Planck equation $\dot{Q}=-\bar{\lambda} \partial_{z} Q+\frac{1}{2} \overline{\lambda^{2}} \partial_{z}^{2} Q$. The latter admits a stationary solution $Q(z) \propto \exp (\beta z)$, and the corresponding $P(y)$ is the same form as (6) with $\beta$ given by (8).

In order to test the validity of our argument, the array of logistic maps (1) has been analyzed numerically. In Fig. 2, we display $P(y)$ versus $y$ for some values of $K$, with $x$ fixed at a sufficiently small value. As expected, 


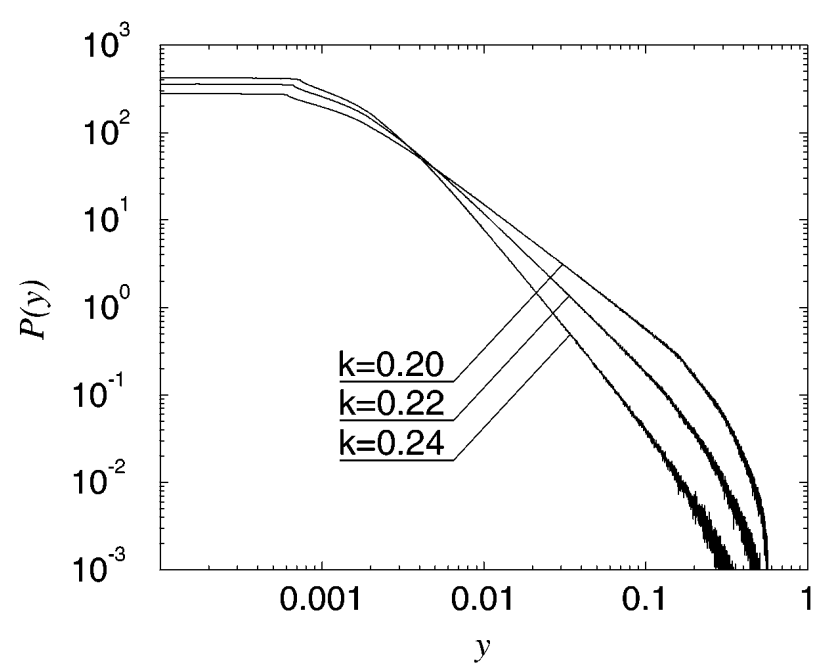

FIG. 2. Probability density $P(y)$ in logarithmic scales for the driven logistic maps (1). For each $K$ value, $P(y)$ obeys a power law in the intermediate range of $y$. The exponent changes as $-1.40,-1.77$, and -2.33 with increasing $K$. $a=3.7(1-K)$, and $x=1024^{-1}$.

$P(y)$ exhibits a power-law dependence on $y$ for not too small or too large $y$, with the exponent depending on $K$. Figure 3 shows moments $\left\langle y(x)^{q}\right\rangle$ versus $x$ for some values of $q$. Their power-law dependence on $x$ is clear, but the observed change of the exponent with $q$, indicated in the small box, is not so sudden across $q=|\beta|$ as the formula (10) predicts. The main source of this discrepancy seems to be the existence of the crossover regime $\mathrm{C} 1$.

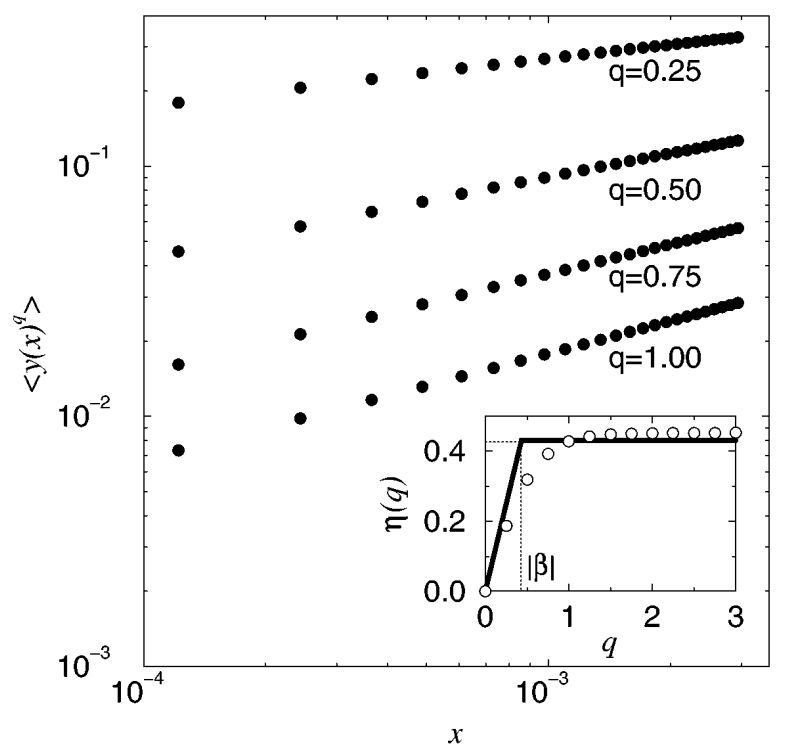

FIG. 3. Moments $\left\langle y(x)^{q}\right\rangle$ vs $x$ in logarithmic scales for the driven logistic maps (1), showing power-law dependence on $x$ for each $q$ with $q$-dependent exponent $\eta(q)$. Numerical $\eta$ vs $q$ curve is displayed in the inset and compared with the theoretical curve. $K=0.2$, and $a=3.7(1-K)$.
Up to this point, we have considered uncoupled units. We now show some evidence that inclusion of shortrange coupling leaves the above-described power-law behavior of moments essentially unchanged. As an illustration, we modify (1) with additional diffusive coupling of the form $D\left\{f\left(X_{n}(j+1)\right)+f\left(X_{n}(j-1)\right)-\right.$ $\left.2 f\left(X_{n}(j)\right)\right\} / 2$. Without the forcing term $h_{n}$, such a model would be identical with the usual coupled map lattice. In Fig. 4, the second moments $\left\langle y(x)^{2}\right\rangle$ are compared between the two systems, one with diffusive coupling $(D=0.1)$ and the other without. The deviation from a power law in the presence of coupling is limited to the range covering ten or so units out of $N(=4096)$. This defines a lower cutoff length $x_{d}$ similar to the dissipation length in fully developed fluid turbulence. Although $x_{d}$ will increase with $D$ like $x_{d} \propto \sqrt{D}$, we have a prefactor $N^{-1}$, so that $x_{d}$ can be made arbitrarily smaller than 1 (i.e., the upper cutoff) by increasing $N$ indefinitely. Thus, the intermediate range of $x$, which is similar to the inertial subrange, has a sufficient extention over which the power-law nature of correlations is practically unaffected.

It is also worth noting that whether the dynamical units involved are themselves chaotic or not is unimportant to the power-law nature of $P(y)$ and $\left\langle y(x)^{q}\right\rangle$. This has been confirmed with driven phase oscillators of the form $\dot{\phi}_{j}=1-c \cos \phi_{j}+h_{j}(t),|c|<1$ with suitable random driving $h_{j}(t)$. To save space, however, we will not show such numerical data here.

Some new aspects of our turbulent field are revealed through an analysis of the differential amplitudes $Y(j)$ or quantities defined similarly when the spatial dimension is two or higher. We call such a field the $Y$ field. The situation is analogous to fully developed fluid turbulence where the study of the energy dissipation field provides rich information which would hardly be available from the study of the velocity field alone. We will restrict

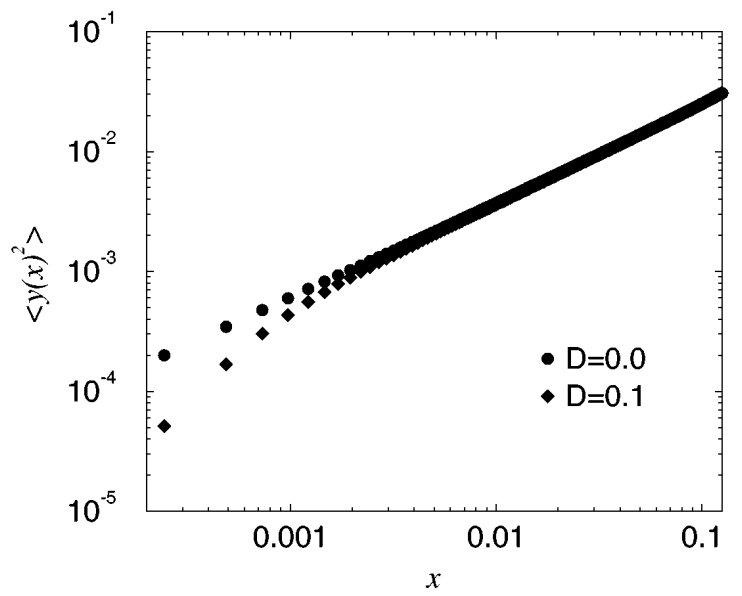

FIG. 4. The second moment $\left\langle y(x)^{2}\right\rangle$ vs $x$ in logarithmic scales for the driven logistic maps (1) with additional diffusive coupling $(D=0.1)$ and without $(D=0) . \quad K=0.2, a=$ $3.7(1-K)$, and $N=4096$. 


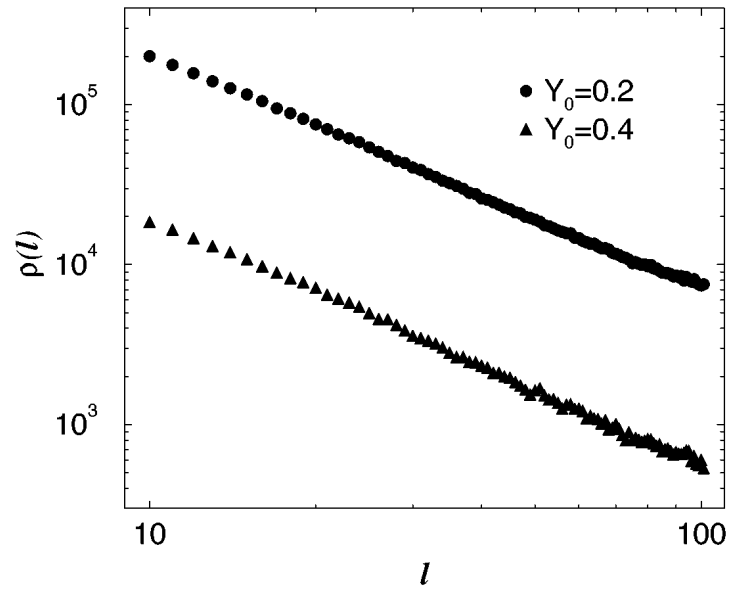

FIG. 5. Size distribution $\rho(l)$ of the laminar domains of the $Y$ field $[\equiv Y(x)=|X(x+\delta)-X(x)| / \delta]$ in logarithmic scales for the driven logistic maps (1). $K=0.2, a=3.7(1-K)$, $N=1024, \delta=N^{-1}, Y_{0}=0.2$, and 0.4 .

our discussion to $1 \mathrm{D}$ systems below. Note that in the absence of short-range coupling a true derivative $d X / d x$ may not exist in the continuum limit, especially when the amplitude profile is fractal. The $Y$ field must then be redefined as $Y(x) \equiv \delta^{-1}|X(x+\delta)-X(x)|$ with finite but sufficiently small $\delta$.

Spatial intermittency of $Y(x)$ as exemplified in Fig. 1(b) may be analyzed similarly to the case of on-off intermittency. This is achieved by measuring the probability density $\rho(l)$ for the space interval $l$ over which the units are in the laminar state, namely, their $Y$ values stay below a certain threshold $Y_{0}$. Such an analysis was done for the driven logistic maps (1). It is clear from Fig. 5 that, as in the on-off intermittency, $\rho(l)$ exhibits an inverse power law. We confirmed that, for not too small or too large $Y_{0}$, the exponent is insensitive to the choice of $Y_{0}$, but depends on $K$.

A more thorough characterization of the $Y$ field is provided by the generalized fractal dimensions $D_{q} \equiv(q-$ $1)^{-1} \lim _{\epsilon \rightarrow 0} \ln \sum_{i} \mu_{i}^{q} / \ln \epsilon$ [7]. Here, the measure $\mu_{i}$ of the $i$ th box of size $\epsilon$ is defined as being proportional to the integral of $Y(x)$ within the same box, with the condition of the total measure being normalized. Similar multifractal analysis was performed for the energy dissipation field of fully developed fluid turbulence [8]. Figure 6 shows $D_{q}$ obtained for the driven logistic maps (1). Note that $D_{0}=1$, which is simply because $Y(x)$ is nonvanishing almost everywhere. In contrast to the power-law behavior of the original amplitude field, which was rather easy to explain, the multifractal nature of the $Y$-field seems nontrivial and remains to be explained. This is because the latter does not result simply from the probability density for a single $y$ variable, but is related to singular correlations among different $y$ 's created by the spatially correlated forcing field.

Finally, we emphasize the importance of the type of turbulence reported here. Because of its remarkable robust-

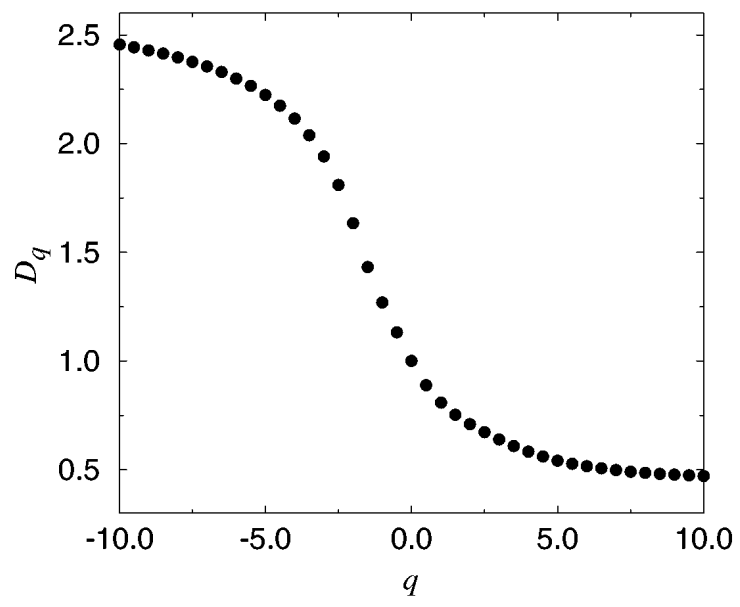

FIG. 6. Dimension spectrum $D_{q}$ of the $Y$ field for the driven logistic maps (1). $K=0.2, a=3.7(1-K), N=1024$, and $\delta=N^{-1}$.

ness, similar phenomena should exist quite universally. They may appear in a wide variety of coupled and uncoupled systems, once placed in a long-wave randomly fluctuating external field. Some possible candidates would be electrohydrodynamic convection in nematic liquid crystals, light-sensitive Belousov-Zhabotinsky reaction, and nonlinear optical media. Their experimental verification is strongly desired.

The authors thank P. Marcq for fruitful discussions and careful reading of the manuscript. The present work has been supported by the Japanese Grant-in-Aid for Science Research Fund from the Ministry of Education, Science and Culture (No. 07243106).

[1] Y. Kuramoto and H. Nakao, Phys. Rev. Lett. 76, 4352 (1996).

[2] L. Yu, E. Ott, and Q. Chen, Phys. Rev. Lett. 65, 2935 (1990).

[3] A. S. Monin and A. M. Yaglom, Statistical Fluid Mechanics (MIT Press, Cambridge, MA, 1975).

[4] A. Schenzle and H. Brand, Phys. Rev. A 20, 1628 (1979).

[5] H. Fujisaka and T. Yamada, Prog. Theor. Phys. 69, 32 (1983); A. S. Pikovsky, Z. Phys. B 55, 149 (1984); L. Pecora and T.L. Carroll, Phys. Rev. Lett. 64, 821 (1990); N. Platt, E. A. Spiegel, and C. Tresser, Phys. Rev. Lett. 70, 279 (1993); J. F. Heagy, N. Platt, and S. M. Hammel, Phys. Rev. E 49, 1140 (1994); S.C. Venkataramani et al., Physica (Amsterdam) 96D, 66 (1996).

[6] A. S. Pikovsky and P. Grassberger, J. Phys. A 24, 4587 (1991); A.S. Pikovsky, Phys. Lett. A 165, 33 (1992); P. Khoury, M. A. Liberman, and A. J. Lichtenberg, Phys. Rev. E 54, 3377 (1996).

[7] E. Ott, Chaos in Dynamical Systems (Cambridge Univ. Press, Cambridge, 1993).

[8] C. Meneveau and K. R. Sreenivasan, Nucl. Phys. B (Proc. Suppl.) 2, 49 (1987). 